\title{
Opportunity or Affection? Participation of \\ Respondents' Partners in a Multi-actor Survey
}

@SAGE

\section{Bettina Müller'}

\begin{abstract}
Multi-actor studies are particularly suited for partner and family research, as they capture relationships beyond the conventional restraint of the household. Previous research on partner participation in the German Family Panel indicates higher participation of cohabiting and married partners compared to those living apart together. The present study evaluates whether this finding is due to unobserved relationship quality aspects associated with relationship status, differentially affecting the likelihood of partner response, or rather to field procedures favoring the participation of coresidential partnerships. Fixed effects models find a positive effect of moving in together on partner response, indicating that part of the relationship status effect found in previous research can, in fact, be attributed to coresidence. Analyzing the response process in detail reveals that the effect of moving in together goes back to main respondents' consent to the partner survey, whereas no significant effect can be found on partner participation.
\end{abstract}

\footnotetext{
' Department of Sociology, LMU Munich, Munich, Germany
}

\section{Corresponding Author:}

Bettina Müller, Department of Sociology, LMU Munich, Konradstraße 6, 8080I Munich, Germany.

Email: bettina.mueller@soziologie.uni-muenchen.de 


\section{Introduction}

Multi-actor studies gather information on individuals with an explicitly defined relationship to one another. Usually within partner and family research, and as in the German Family Panel pairfam, a multi-actor approach is implemented by including partners and family members of an originally sampled primary respondent into the survey as so-called secondary respondents (Kalmijn and Liefbroer 2011; Pasteels 2015). One major advantage of multi-actor over household designs is that they are not restricted to relationships between persons living together. Additionally, information on subjective characteristics such as personal values, feelings, and attitudes can be gathered directly rather than by proxy reports from primary respondents. A drawback of multi-actor data, however, is the substantial level of nonresponse and possible selectivity bias (Havermans et al. 2014; Kalmijn and Liefbroer 2011; Schröder et al. 2013).

Selective nonresponse in multi-actor surveys has been evaluated with a focus on the specific survey design, in contrast to conventional population samples (Kalmijn and Liefbroer 2011; Schröder et al. 2013). Secondary respondent participation requires the consent of primary respondents (anchors) and is therefore thought to depend, in part, on the relationship quality between anchors and secondary respondents. The analysis of partner participation in the first pairfam wave (Schröder et al. 2013) did not reveal clear evidence about this but did show a strong effect of relationship status: Cohabiting and married partners were more likely to participate than those living apart together (LAT).

Cohabiting and married statuses represent increased institutionalization and commitment between partners and have been found to be associated with further relationship quality aspects such as higher relationship happiness and stability (Brown 2004; Dorbritz and Naderi 2012). The effect of relationship status could thus indicate selectivity toward more committed relationships and, possibly, further unmeasured relationship quality aspects. However, relationship status is also linked to a couple's living arrangement (i.e., whether they live apart or together). When compared to LAT constellations, coresidence provides increased opportunities for partners to be presented with the study, favoring partner participation. Additionally, field procedures imply that the paper transfer of the questionnaire is easier if the couple lives at the same address.

The purpose of this study is to reevaluate the effect of relationship status on partner participation found by Schröder et al. (2013) in more detail, expanding to wave 6 of the pairfam study. More specifically, it has not yet 
been determined whether varying relationship statuses mirror different relationship quality aspects (e.g., levels of commitment), which might differentially affect the likelihood of partner response, or whether it is the fact that certain relationship statuses imply coresidence that favors participation. It is, however, crucial to determine whether multi-actor data suffer from selectivity regarding relationship quality, or whether survey design features favor the participation of specific respondent groups.

Knowledge of selectivity issues, especially those concerning pairfam's core variables, is important from a data user's perspective and provides information for corrections of nonresponse bias. If, however, an underrepresentation of LAT constellations arises from field procedures, this can and should be addressed in future multi-actor designs. To disentangle these two issues, I make use of a panel framework with multi-actor data to analyze the effect of moving in together with fixed effects regression models, comparing partner response of the same anchor-partner dyads before and after the move. Further, I evaluate partner response at both stages of the data collection process separately: anchor consent to the partner interview and partner participation (provided there is anchor consent).

\section{Determinants of Partner Response}

Relationship quality is hypothesized to be a relevant determinant of secondary participation. Low relationship quality (e.g., discontent with the relationship, frequent conflicts, or relationship instability) might decrease anchors' willingness to consent to surveying their partners and family members as this might expose sensitive information. For secondary respondents, providing information about a troubled relationship might also be difficult, and participating in the survey could be seen as doing the anchor a favor, decreasing the likelihood of participation (Kalmijn and Liefbroer 2011; Schröder et al. 2013). Kalmijn and Liefbroer (2011) offer some evidence about this from the Netherlands Kinship Panel Study. Relationship quality, frequency of contact, and support, among other factors, were associated with anchor consent as well as secondary child participation, indicating selectivity toward higher quality and closer relationships. Similarly, in the Divorce in Flanders study, frequent and open communication was revealed to be related to both increased parental consent and child participation (Havermans et al. 2014).

As for pairfam, Schröder et al. (2013) found relationship quality between anchors and their partners to be of little to no importance for partner participation in the first wave, whereas a strong effect was found for 
relationship status. Their analysis included several relationship quality characteristics such as relationship satisfaction, stability, and frequency of conflicts. However, given the measurement difficulties associated with such variables, as well as the likelihood that not all relevant relationship quality aspects have been measured, selection effects may still be at work. The effect of relationship status could thus be partly explained by unobserved relationship quality aspects that favor more institutionalized relationships and that might also correlate with participation in the partner survey (e.g., higher levels of long-term commitment, self-identification with the relationship, joint decision-making processes, etc.). In this case, relationship status can be understood as an indicator of these underlying factors.

Further implications of relationship status for partner response emerge when considering couples' living arrangements. Opportunities to be presented with the pairfam study and to exchange respective information and attitudes are increased for coresiding as compared to LAT couples. If a couple lives together, it is more likely that the partner has been addressed by the interviewer either at the contact stage of the anchor interview or even during said interview. Interviewers play an important role in gaining respondents' cooperation, for example, by providing information about the study (Groves and Couper 1998:192). Therefore, contact with the interviewer is thought to increase trust and willingness of partners to participate. Furthermore, the questionnaire transfer is easier: It can either be left at the household or handed over directly, if the partner is present at the time of the anchor interview, as opposed to the anchor delivering the questionnaire or providing the partner's address.

Partner presence at the anchor interview can also be considered crucial for anchors' consent to the partner survey. Uncertainty about their partner's willingness to participate could prevent anchors from consenting, whereas possible concerns can be cleared directly if the partner is present. Both marital and nonmarital coresidence are therefore assumed to positively affect partner response at both stages of the data collection process. From a survey design perspective, relationship status thus matters in terms of whether the couple is coresiding and the likelihood of the partners' presence at the time of the anchor interview.

To summarize, one explanation for the strong effect of relationship status on partner response found by Schröder et al. (2013) is that the association is due to unobserved relationship quality aspects (e.g., long-term commitment, joint decision-making processes), which increase the probability of living together and being married as well as the likelihood of partner 
response. If this holds true, when additionally accounting for all stable components of these unobserved characteristics in a within-person estimation, the effect of moving in together should be negligible. If, on the other hand, moving in together is shown to have a positive effect on partner response, this would indicate that coresidence is an important contributing factor in terms of field procedures favoring participation, rather than relationship quality. The effect should then become less substantial when controlling for field-related influences such as partner presence during the anchor interview.

\section{Data and Method}

\section{The German Family Panel pairfam}

Analyses rely on data from the first six waves of the German Family Panel pairfam (Brüderl, Hank, et al. 2015), an annual survey of a random sample of German residents from three birth cohorts: 1971-1973, 1981-1983, and 1991-1993. Data have been collected with computer-assisted personal interviews, starting in 2008 with approximately 4,000 interviews from each cohort. The focus of the panel study is on partnership dynamics, generative behavior, parenting and child development, and intergenerational relationships (for more details, see Huinink et al. 2011).

The multi-actor approach implemented in pairfam foresees the inclusion of the anchor respondent's partner, parents, and children into the survey. Integrating the partners is organized as follows: Beginning with wave 1, all anchors reporting to be currently in a relationship are asked for permission to interview their (resident or nonresident) partner approximately halfway through their interview. If consent is granted, partners receive a separate introduction letter and a paper-and-pencil questionnaire including a postpaid return envelope that - by choice of the anchor-are either left at the anchor's household or sent to the partner's address, as provided by the anchor. The questionnaire can then either be mailed back or collected by the interviewer. If the partner is present in the household at the time of the anchor interview, the questionnaire can be handed off directly and collected immediately if completed by the end of the anchor interview. In the case of separation, former partners are no longer surveyed, while new partners, if any, are included into the survey.

Partner response rates are quite low, as is common in multi-actor surveys (Bastaits et al. 2015; Dykstra et al. 2012): Averaged over the first six waves, response rates amount to $49.3 \%$. Thus, only half of the potential 
anchor-partner dyads are available for analysis. Of all partnered anchors, $68.4 \%$ agreed to the partner survey. Of those, $72.1 \%$ of their partners returned the questionnaire (for more details on partner response rates, see Brüderl, Schmiedeberg, et al. 2015).

\section{Panel Analysis of Partner Response}

Associations between relationship status and partner response observed with cross-sectional data might be overestimated due to selection processes: Unobserved relationship characteristics might increase the probability of living together and being married as well as partner response. Thus, one major advantage of a panel framework with multi-actor data is that partner response can be analyzed longitudinally, applying fixed effects panel regression. Using only within-person variation, effects can be estimated while accounting for all such stable, unobserved selection factors (for details on fixed effects regression, see, e.g., Allison 2009; Brüderl and Ludwig 2015). To evaluate the importance of relationship quality and field procedures for partner response, I differentiate between LAT and coresidence (married and nonmarried). My focus lies on the causal effect of moving in together on partner response, comparing individual changes in response probabilities before and after the move.

The effect of moving in together is first analyzed for overall partner response probability (i.e., whether a partner interview is realized for anchors with a partner). Partner response is then further evaluated at both stages of the response process separately: anchor consent and partner participation (provided anchor consent). The fixed effects regressions are based on linear probability models, which provide a simple interpretation of results. Further, for the sake of comprehensibility, I present average marginal effects computed for each observation, given their respective values on other variables and then averaged over all observations. Standard errors are adjusted for clustering by ID number.

\section{Estimation Samples for Fixed Effects Analyses}

Analyses are based on two estimation samples: sample 1 is defined to analyze overall partner response and anchor consent and sample 2 to analyze partner participation of those anchors from sample 1 who consented to the partner interview. Both samples include observations of respondents who reported having a partner, both individuals at least 18 years old ( $N=27,507$ observations), and may contain observations for different 
relationships for one given anchor. Furthermore, fixed effects analysis requires at least two observations per respondent and an analysis sample defined with regard to the event under study (Brüderl and Ludwig 2015:346). Both samples are therefore further restricted to respondents with at least two observations with the same partner $(N=23,461)$, who are at risk of moving in together within the observation period (i.e., who are LAT when first observed) and either remain LAT or then coreside. LAT married respondents $(N=107)$ have been excluded from the analysis. Given these restrictions, sample 1 contains 1,923 anchor respondents and 6,230 personyear observations with a total of 856 anchors who experienced a change from LAT to coresidence within the observation period. Sample 2 includes 3,019 observations from 977 anchors and 488 status changes.

It should be noted that the sample restrictions necessary for fixed effects analyses may limit the generalizability of findings but ultimately allow for a more precise test of the effect of living together on partner response.

\section{Models}

Models include anchor characteristics, information on their current relationship, and proxy information on partner characteristics available from the anchor interview, irrespective of whether the partner participated.

In a fixed effects panel analysis, common time-constant determinants of survey participation such as migration status (for an overview, see Watson and Wooden 2009) are controlled for by design, placing the focus on timevarying control variables. Baseline models for overall partner response, anchor consent, and partner participation include only time-varying factors that are not affected by moving in together. Age and relationship duration are thought to be linked to both the likelihood of living together and partner response. Partners' age is included into the model for overall partner response and partner participation and anchors' age to the anchor consent model. To account for a decreasing influence with each additional year, relationship duration is included in its logarithmized form (Schröder et al. 2013).

All models control for whether a particular observation concerns the anchors' first, second, or third partnership within the observation period. Additionally, partners' employment status is controlled for, as it is assumed to affect partner participation via time constraints as well as the anchor's estimation of their partner's willingness to participate. Further, anchors' own interview experience is hypothesized to affect the likelihood of consent to interview secondary respondents (Kalmijn and Liefbroer 2011) and is 
operationalized as information on item nonresponse in the anchor interview (Loosveldt et al. 2002). Models explaining overall partner response and anchor consent probability additionally include the percentage of item nonresponse and nonresponse for household income and intimate questions ("I don't know" and "no answer" to at least one of the questions on satisfaction with sex life and/or use of contraceptives).

Next, my focus lies on the importance of relationship quality and field aspects at both stages of data collection. To this end, relationship characteristics and field procedure variables are then added to the models explaining anchor consent and partner participation probability. The choice of relationship characteristics closely follows Schröder et al. (2013) but is restricted to those measured in all of the first six pairfam waves: marriage, having children, anchor reports on relationship satisfaction, several adapted dimensions of the Network of Relationship Inventory (NRI; Furman and Buhrmester 1985), and relationship stability (see Thönnissen et al. 2015). Relationship satisfaction is measured on an 11-point scale $(0=$ very dissatisfied, $10=$ very satisfied), whereas dummy variables for conflicts and intimacy in the partnership, appreciation by and dominance of the partner $(0=$ no, $1=$ yes $)$ have been created based on eight items of the NRI. The variable "relationship instability" indicates the number of relationship instability scale items ("thought relationship was in trouble," "thought about separation," and "proposed separation") to which the anchor answered affirmatively (0-3).

As for field procedures, information on partner presence during the anchor interview is of particular interest for both anchor consent and partner participation. Additionally, the mode of questionnaire transfer is included into the model explaining partner participation. This variable differentiates between anchors who preferred a personal transfer (if their partner was present in the household at the time of the anchor interview) and those who preferred the questionnaire to be left behind or sent to their partner's address.

Descriptive statistics of the variables in both estimation samples can be found in the Online Appendix (Table S1).

\section{Results}

To examine if the effect of relationship status on overall partner response found in wave 1 (Schröder et al. 2013) holds when extending the analysis sample to wave 6 and differentiating between anchor consent and partner participation, I first ran logistic regressions based on pooled data. 


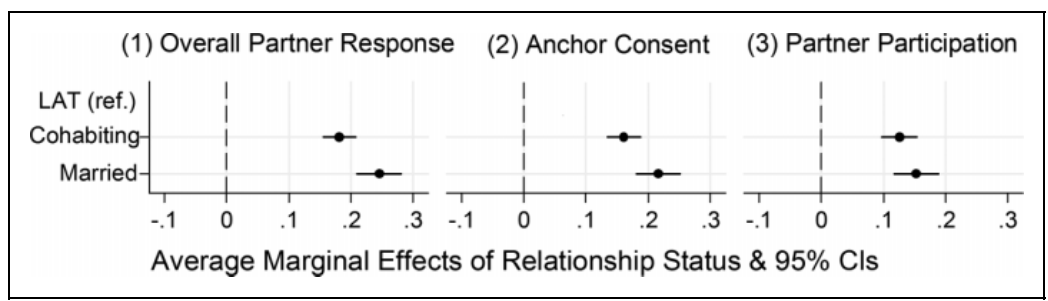

Figure I. Effects of relationship status, pooled logistic regressions. Note: Models include relationship characteristics, partner employment status, partner presence, number of relationships, wave dummies (all models), item nonresponse, anchor sociodemography (models I and 2), and partner sociodemography (models I and 3); sample of anchors with partner (both at least I8), waves I-6; model I: $N=24$, II (7,8I9 anchors); model 2: $N=24,740$; and model 3: $N=$ 17,665 .

Controlling for common sociodemographic influences on response (e.g., age, migration status) and relationship characteristics (Online Appendix Table S1), cohabiting and being married have a pronounced positive effect on overall response, anchor consent, and partner participation probability as compared to LAT constellations (Figure 1).

Turning to the fixed effects models (Figure 2), moving in together has a pronounced and highly significant effect on overall partner response and anchor consent probability, suggesting that both change as the couple moves in together. Considering that estimates are based on withinperson changes only, the average magnitude of this effect appears to be important, with the likelihood of overall response increased by approximately 8 , anchor consent by approximately 11 percentage points. Results for partner participation show a small positive but not significant effect of moving in together. At this stage of the response process, the effect of relationship status found in the pooled logistic regression model (Figure 1) thus appears to be due to selection (i.e., nonrandom cohabitation of couples).

To evaluate the underlying causes of the association between moving in together and partner response and the importance of relationship quality and field aspects on both data collection stages, I add relationship (models $5 \mathrm{a}$ and $6 \mathrm{a}$ ) and field characteristics (models $5 \mathrm{~b}$ and $6 \mathrm{~b}$ ) to the models explaining anchor consent and partner participation, respectively. The complete results can be found in the Online Appendix (Tables S2 and S3, respectively). 


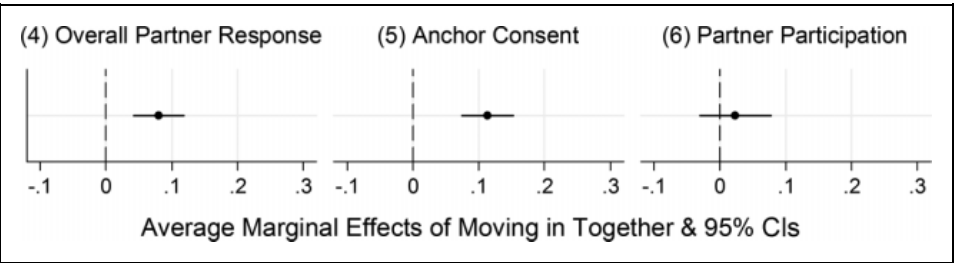

Figure 2. Effects of moving in together, linear probability fixed effects regressions. Note: Models include age, relationship duration, number of relationships, partner employment status (all models), and item nonresponse (models 4 and 5); waves I-6; model 4: $N=5,927$ (I,855 anchors); model 5: $N=6,093$; and model 6: $N=2,963$.

Concerning anchor consent, relationship quality characteristics including relationship satisfaction, stability, and NRI variables appear to be of little importance. None of these indicators substantially alter the effect of moving in together in the baseline model. Next, additional information on partner presence during the anchor interview is included, which has a strong positive effect on anchor consent probability (16.5 percentage points). The effect of moving in together remains stable; thus, living together does not appear to affect anchor consent via partner presence at the anchor interview. However, increased opportunities to exchange information and to evaluate partners' attitudes about the study might still be a reasonable explanation, although this cannot be further explored here, as no additional information on this aspect was collected. For example, if the partner was at home at the time of the anchor interview though not in the same room, this is not considered in the partner presence variable but would provide the opportunity for consultation just the same.

At the partner participation stage, relationship aspects appear to be more relevant, with changes toward relationship instability (i.e., more instability items answered affirmatively) negatively associated with the likelihood of partner participation (6.4-10.8 percentage points). Both partner presence and the immediate transfer of the partner questionnaire at the anchor interview have a highly significant positive effect on partner participation (18.5 and 9.3 percentage points). The effect of partner presence persists when including information on the questionnaire transfer, indicating that partner presence is not only important in terms of questionnaire transfer (and return), but also that contact with the interviewer is favorable for partner cooperation.

In summary, there is evidence that part of the relationship status effect observed in the pooled logistic regression models can, in fact, be explained 
by unobserved heterogeneity due to selection, as in the fixed effects estimations moving in together has no effect at the partner participation stage. Here, observed and unobserved relationship aspects seem to be more relevant. The effect of relationship status on anchor consent, however, appears to evolve from coresidence: While controlling for observed and unobserved relationship characteristics, anchor consent probability increases significantly as the couple moves in together.

\section{Discussion}

In a relationship and family study, it is of great importance to discern whether all relationship types can be adequately represented. Previous research suggests lower partner response among less institutionalized relationships (Schröder et al. 2013), but it is not clear why including these relationships is more challenging. This study yields evidence that the effect of relationship status on partner response can't be explained only by selective participation concerning relationship quality aspects, but that it is also at least partly due to living arrangements.

The hypothesized explanation for this result-increased opportunities of partner exposure to the study and to exchange respective information and attitudes after the move-however, can't be fully evaluated with the available field variables. Additional information on this aspect (e.g., whether the couple discussed partner participation) was not collected. It is also worth mentioning that although all relevant relationship characteristics included in the data set have been considered, unobserved time-varying characteristics could still be involved, affecting both the likelihood of moving in together and partner response. For example, the effect of moving in together might partly be explained by unmeasured changes in the perceived institutionalization of the relationship and commitment before the move (e.g., due to the decision itself to move in together). Finally, it cannot be ruled out that some institutional aspects of cohabitation and marriage that do not relate to relationship quality affect partner response (e.g., the implicit commitment to deal with "tasks" like addressing a partner survey request as a couple).

Including LAT relationships into partner and family research is obviously challenging, whether this is due to specific field procedures or relationship characteristics. As extending the household perspective is one of the main goals of a multi-actor approach, particular attention should be paid to the inclusion of LAT constellations in future multi-actor designs. In this regard, results from the two-stage analysis of partner response offer 
practical implications for data collection: In a within-person estimation, partner presence during the anchor interview revealed to be an important determinant of both anchor consent and partner response. For anchors, opportunities for consultation with their partner appear to be relevant; for partners, contact with the interviewer seems crucial.

\section{Acknowledgment}

This article uses data from the German Family Panel (pairfam) coordinated by Josef Brüderl, Karsten Hank, Johannes Huinink, Bernhard Nauck, Franz Neyer, and Sabine Walper. pairfam is funded as a long-term project by the German Research Foundation (DFG).

\section{Declaration of Conflicting Interests}

The author(s) declared no potential conflicts of interest with respect to the research, authorship, and/or publication of this article.

\section{Funding}

The author(s) received no financial support for the research, authorship, and/or publication of this article.

\section{Supplemental Material}

The online appendices are available at http://journals.sagepub.com/doi/suppl/ $10.1177 / 1525822 X 16671702$.

\section{References}

Allison, P. D. 2009. Fixed effects regression models. Thousand Oaks, CA: Sage.

Bastaits, K., I. Pasteels, K. Ponnet, and D. Mortelmans. 2015. Adult non-response bias from a child perspective. Using child reports to estimate father's nonresponse. Social Science Research 49:31-41.

Brown, S. L. 2004. Moving from cohabitation to marriage: Effects on relationship quality. Social Science Research 33:1-19.

Brüderl, J., K. Hank, J. Huinink, B. Nauck, F. J. Neyer, S. Walper, P. Alt, P. Buhr, L. Castiglioni, C. Finn, K. Hajek, M. Herzig, B. Huyer-May, R. Lenke, B. Müller, T. Peter, V. Salzburger, C. Schmiedeberg, E. Schubach, P. Schütze, N. Schumann, C. Thönnissen, and B. Wilhelm. 2015. The German family panel (pairfam). GESIS Data Archive, Cologne, Germany. ZA5678, data file version 6.0.0. doi: 10.4232/pairfam.5678.6.0.0. 
Brüderl, J., and V. Ludwig. 2015. Fixed-effects panel regression. In The Sage handbook of regression analysis and causal inference, eds. H. Best and C. Wolf, 327-57. London: Sage.

Brüderl, J., C. Schmiedeberg, L. Castiglioni, O. Arránz Becker, P. Buhr, D. Fuß, V. Ludwig, J. Schröder, and N. Schumann. 2015. The German family panel: Study design and cumulated field report (Waves 1 to 6 ). http://www.pairfam.de/filead min/user_upload/redakteur/publis/Dokumentation/TechnicalPapers/TP01_ Field-Report_pairfam6.0.pdf (accessed April 19, 2016).

Dorbritz, J., and R. Naderi. 2012. Stability of bilocal relationships - Conditions and development paths. An analysis of the first and second wave of pairfam. Comparative Population Studies 37:429-62.

Dykstra, P. A., M. Kalmijn, T. C. M. Knijn, A. E. Komter, A. C. Liefbroer, and C. H. Mulder. 2012. Codebook of the Netherlands Kinship Panel Study, a multi-actor, multi-method panel study on solidarity in family relationships, Wave 2. NKPS Working Paper No. 8. Netherlands Interdisciplinary Demographic Institute, The Hague, the Netherlands. http://www.nkps.nl/CodeBook/CodeBookFrame.htm (accessed April 19, 2016).

Furman, W., and D. Buhrmester. 1985. Children's perceptions of the personal relationships in their social networks. Developmental Psychology 21:1016-24.

Groves, R., and M. Couper. 1998. Nonresponse in household interview surveys. New York: John Wiley.

Havermans, N., S. Vanassche, and K. Matthijs. 2014. Methodological challenges of including children in family research: Measurement equivalence, selection bias and social desirability. Child Indicators Research 8:975-97.

Huinink, J., J. Brüderl, B. Nauck, S. Walper, L. Castiglioni, and M. Feldhaus. 2011. Panel analysis of intimate relationships and family dynamics (pairfam): Conceptual framework and design. Zeitschrift für Familienforschung 23:77-100.

Kalmijn, M., and A. C. Liefbroer. 2011. Nonresponse of secondary respondents in multi-actor surveys: Determinants, consequences, and possible remedies. Journal of Family Issues 32:735-66.

Loosveldt, G., J. Pickery, and J. Billiet. 2002. Item nonresponse as a predictor of unit nonresponse in a panel survey. Journal of Official Statistics 18:545-57.

Pasteels, I. 2015. How to weight data with a dyadic multi-actor design. Survey Insights: Methods from the Field. Weighting: Practical Issues and "How to" Approach. http://surveyinsights.org/?p=5127 (accessed April 19, 2016).

Schröder, J., L. Castiglioni, J. Brüderl, and U. Krieger. 2013. The influence of relationship quality on the participation of secondary respondents: Results from the German Family Panel. Comparative Population Studies 37:591-614. 
Thönnissen, C., B. Wilhelm, S. Fiedrich, P. Alt, and S. Walper. 2015. Scales manual, Waves 1 to 6. http://www.pairfam.de/fileadmin/user_upload/redak teur/publis/Dokumentation/Manuals/Scales_Manual_pairfam_6.0.pdf (accessed April 19, 2016).

Watson, N., and M. Wooden. 2009. Identifying factors affecting longitudinal survey response. In Methodology of longitudinal surveys, ed. P. Lynn, 157-81. Chichester, UK: Wiley. 